Casos Clínicos

Arch. Esp. Urol., 60, 10 (1.200-1.203), 2007

\section{TUMOR FIBROSO SOLITARIO INGUINAL MALIGNO. APORTACIÓN DE UN CASO}

\author{
P. Garrido Abad, A. Coloma Del Peso, L. M. Herranz \\ Fernández, M. Jiménez Gálvez, G. Bocardo Fajardo, \\ R. Arellano Gañán, I. Pereira Sanz y T. Reina Durán'.
}

Servicio de Urología del Hospital de La Princesa. Madrid. Servicio de Anatomía Patológica'. Hospital de La Princesa. Madrid. España.

Resumen.- OBJETIVO: El Tumor Fibroso Solitario (TFS) es una neoplasia poco frecuente que fue descrita por primera vez en la pleura, pero puede aparecer en diferentes localizaciones. Su presencia en la zona inguinal es extremadamente rara.

MÉTODO/RESULTADOS: Presentamos el caso de un varón de 74 años con masa inguinoescrotal derecha de 50 años de evolución. Tras extirpación quirúrgica de la misma es diagnosticado de tumor fibroso solitario.

Pablo Garrido Abad

Santiago Bernabeu, 4 5 Pta. 4

28036 Madrid. (España)

pgabad@hotmail.com

Trabajo recibido: 23 de febrero 2007
CONCLUSIONES: La inmensa mayoría de los tumores fibrosos solitarios se comportan de manera benigna. Un pequeño porcentaje se comporta de modo más agresivo, con recurrencia local y metástasis. Su tratamiento es eminentemente quirúrgico, pero después debemos realizar seguimiento a largo plazo en todos los casos.

Palabras clave: Tumor fibroso solitario. Partes blandas. CD 34.

Summary.- OBJECTIVE: Solitary fibrous tumours (SFTS) are rare neoplasias that were first reported in pleura, but can occur in different sites. Inguinal location is extremely rare.

METHODS/RESULTS: We report one case of a 74 year old man with a right inguinoscrotal mass with 50 years of evolution. After surgical extirpation, solitary fibrous tumour was diagno-sed.

CONCLUSIONS: The vast majority of solitary fibrous tumours (SFTs) have a benign course. Only a few cases have aggressive behaviour, with local recurrence and metastasis. Treatment is surgical, but we must do a long-term follow up in all cases.

Keywords: Solitary fibrous tumour. Soft tissues. CD 34.

\section{INTRODUCCIÓN}

El Tumor Fibroso Solitario (TFS) es una neoplasia fusocelular poco frecuente, que posiblemente deriva de fibroblastos y células mesenquimales (1).

Fue inicialmente descrito en la pleura en 1931 por Klemperer y Rabin (2) y durante mucho tiempo se consideró ésta su única localización. A lo largo de la historia el TFS ha recibido múltiples denominaciones: mesotelioma fibroso, tumor fibroso localizado, mesotelioma localizado, fibroma pleural. En la actualidad se han descrito múltiples casos de TFS en otras localizaciones.

Se trata de un tumor de tamaño variable con un patrón histológico atípico (alternancia hiper e hipocelular con arquitectura vascular hemangiopericitoide en la mayoría de los casos) y positividad inmunohistoquímica en más del $90 \%$ para CD34.

Las lesiones suelen ser asintomáticas y su diagnóstico incidental en la mayoría de casos. Su comportamiento es generalmente benigno. Aproximadamente un 10\% de los casos de TFS extratorácico pueden recurrir localmente o a distancia (3). Todavía permanecen en controversia los factores que predicen su comportamiento maligno. 
El tratamiento indicado es siempre la extirpación quirúrgica, y es necesario el seguimiento a largo plazo en todos los casos.

\section{CASO CLÍNICO}

Presentamos el caso de un varón de 74 años de edad que acude a nuestro hospital refiriendo abultamiento y dolor en región inguinal de 50 años de evolución, con crecimiento importante en los últimos seis meses, tras haber permanecido estable durante todo este tiempo.

A la exploración observamos una masa inguinoescrotal derecha de grandes dimensiones y consistencia variable, no adherida a planos profundos. El teste derecho y el cordón espermático se encuentran desplazados, pero independientes de la tumoración.

Se realizan diversas pruebas de imagen. En ecografía se informa como imagen nodular hipoecoica, con una ecogenicidad heterogénea en su interior con áreas hipoecoicas nodulares y bandas hiperecogénicas, así como una zona sin flujo vascular en estudio doppler que sugiere necrosis.

Posteriormente se realizan TAC y RM donde se describe tumoración sólida que ocupa el canal inguinal y saco escrotal derecho de contorno bien definido y una densidad heterogénea en su interior, presencia de necrosis y calcificaciones de aproximadamente $15 \mathrm{cms}$. (Figuras 1 y 5$).$

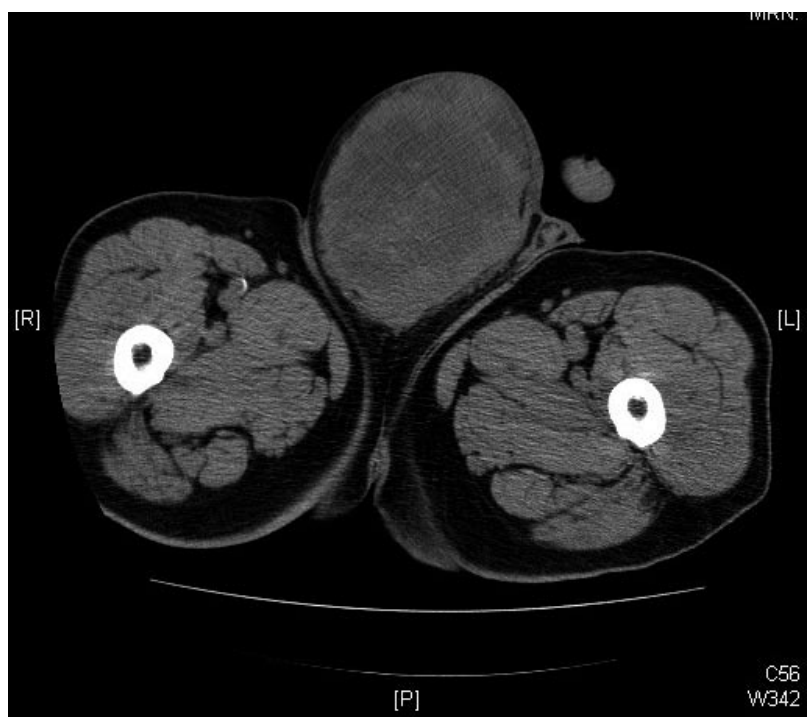

FIGURA 1. Imagen de TAC (sección transversal a nivel inguinoescrotall donde se observa masa heterogénea de aproximadamente $18 \mathrm{cms}$ de diámetro.

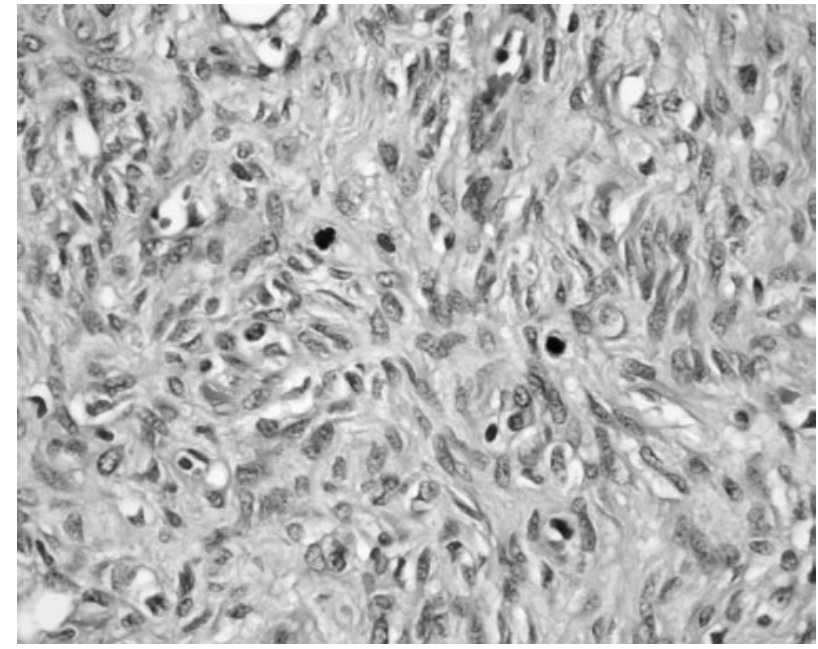

FIGURA 2. Imagen al microscopio de tejido del TFS donde se observa un índice mitótico aumentado, señalado con flechas. (tinción con hematoxilina-eosina, con un aumento 40X).

Se realiza intervención quirúrgica hallándose masa de partes blandas por encima de la aponeurosis de la musculatura abdominal. La masa desplaza el cordón espermático y el testículo sin infiltrarlos, y está bien delimitada. Se extirpa en su totalidad.

En el estudio de anatomía patológica se describe macroscópicamente como tumoración multinodular de 18 cms de diámetro máximo, bien delimitado periféricamente y parcialmente recubierta por fascia, que presenta áreas blanquecinas de aspecto fasciculado con áreas

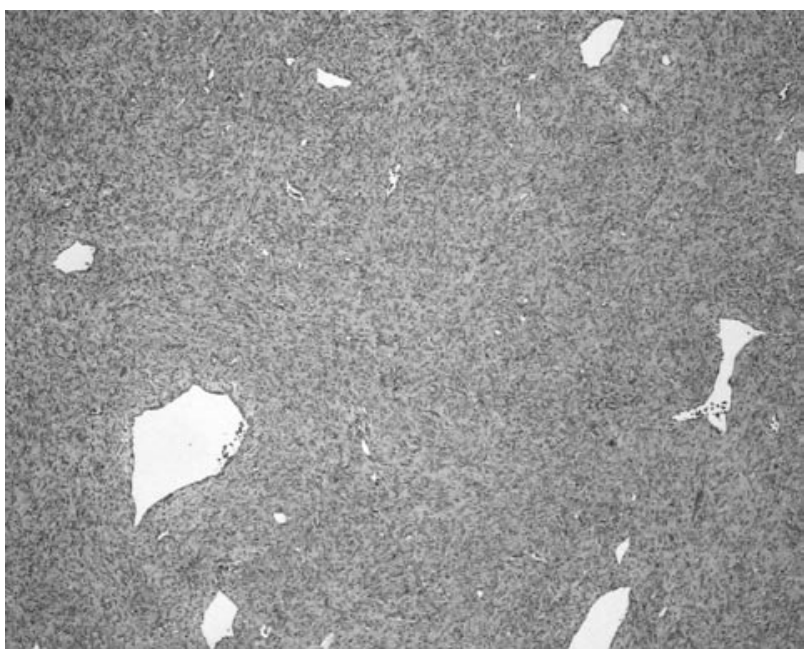

FIGURA 3. Imagen al microscopio de tejido de TFS donde se observa típico patrón vascular hemangiopericitoide, señalado con flechas. (tinción con hematoxilina-eosina). 


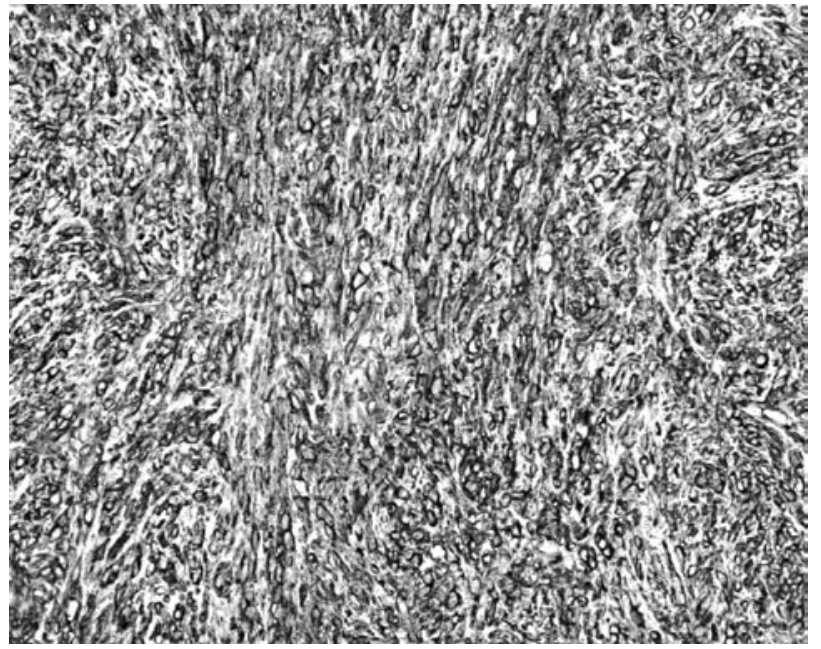

FIGURA 4. Imagen de tejido de TFS donde se aprecia positividad para CD34, con técnicas

inmunohistoquímicas.

geográficas y zonas de necrosis. En examen microscópico se describe alternancia de áreas hipercelulares (con células fusiformes que en algunos casos presentan en el núcleo marcada atipia citológica, patrón hemangiopericitoide y frecuentes mitosis), e hipocelulares (con fibrosis colágena hialina con áreas de degeneración quística). En el seno de la tumoración se observan áreas de necrosis. Se realiza estudio inmunohistoquímico en el que se observa marcada positividad frente a CD 34 y un índice de proliferación cercano al $10 \%$ de la celularidad tumoral medido como positividad nuclear con Mibl. La celularidad tumoral ha resultado negativa frente a HMB45, actina de músculo liso, actina, desmina, S100, CD177, CD31, AE 1-AE3, colágeno 4, citoqueratina de alto peso molecular y receptor de estrógenos (Figuras $2,3,4)$.

El diagnóstico anatomopatológico definitivo es de tumor fibroso solitario maligno.

\section{DISCUSIÓN}

Se ha debatido ampliamente acerca del origen del TFS. Antiguamente se pensó en un origen mesotelial, pero en la actualidad parece haber consenso acerca de su origen mesenquimatoso, probablemente (mio)fibroblástico. $(1,4)$.

Se ha presentado por igual en ambos sexos, y en un amplio espectro de edades, con mayor incidencia entre la $6^{\underline{a}}$ y $7^{a}$ décadas de la vida. Las lesiones son con frecuencia asintomáticas y suelen ser descubiertas incidentalmente. En algunos casos se asocia con sintomatología local debido a afectación de estructuras vecinas, sin embargo la presencia de sintomatología sistémica (hipoglucemia, artralgia, acropaquia) es poco común y

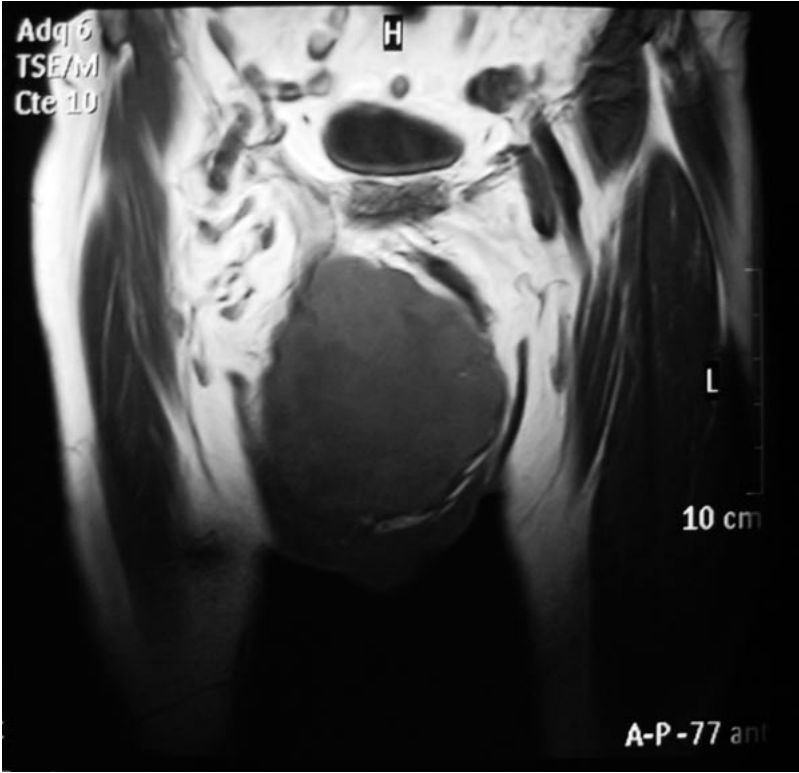

FIGURA 5. Imagen de RMN en corte coronal donde se observa masa de gran tamaño en región inguinoescrotal derecha.

se presenta en menos del $5 \%$ de los casos, con mayor frecuencia en los TFS extratorácicos $(3,5)$. En un principio se diagnosticó exclusivamente en pleura. Actualmente el $60 \%$ de los casos se describen en pleura, formando una importante masa en hemitórax ipsilateral, pero esta neoplasia ha sido ya también descrita en muchas otras localizaciones: menínges, órbita, senos paranasales, tracto respiratorio superior, tiroides, glándula sublingual, pulmón, mediastino, pericardio, tracto gastrointestinal, hígado, peritoneo, retroperitoneo/pelvis glándula adrenal, ovario, vagina, cérvix uterino. En cuanto a sus localizaciones urológicas se han descrito también en vejiga, riñón, próstata, vesículas seminales, cordón espermático y escroto $(6,7)$. Recientemente ha sido descrito también en localización paratesticular (túnica vaginal testicular) (8). En la literatura la localización inguinal es extremadamente infrecuente, con menos de 5 casos descritos (9).

Estos tumores están, por lo general, encapsulados y bien delimitados. En su interior se pueden observar zonas hemorrágicas, degeneración mixoide/quística, necrosis y calcificación focal. La histología típica de este tumor es variable y alterna áreas hipercelulares (proliferación de células fusiformes) con otras hipocelulares (fibrosis colágena hialina con áreas de degeneración quística). Debido a esta variabilidad de patrones histológicos que puede mostrar se ha definido en algunos casos como patrón sin patrón (patternless pattern), y habría que distinguirlo de otros tumores que lo pueden presentar como hemangiopericitoma lipomatoso (con similar patrón vascular pericítico ) y neoplasias fusocelulares (fibromixolipoma dendrítico, angiofibroma de células gigantes, 
lipoma fusocelular). Inmunohistoquímicamente destaca por la positividad de las células fusiformes constituyentes del tumor para CD34 en un $90-95 \%$ de los casos, y para CD99 y bcl-2 en más del 50\%; presentando negatividad para citokeratina, proteína $\$ 100$ y actina/desmina $(6,10,11)$.

El TFS tiende a seguir un curso benigno, incluso algunos autores no reconocen en sus series un comportamiento maligno (12). Sin embargo en otras series se ha documentado comportamiento maligno con una incidencia estimada entre el $10-15 \%(4,13)$, llegando hasta el $23 \%$ en algunas series de TFS extratorácico (12). Clínicamente, su comportamiento no puede ser predecido sólo a partir de los hallazgos histomorfológicos $(3,14,15)$. Criterios de malignidad para esta neoplasia incluyen: Elevado tamaño, difícil resecabilidad, celularidad alta, actividad mitótica (>4 mitosis/10CGA), positividad difusa del p53, elevada expresión de Ki 67 y CD 31, pleomorfismo nuclear y necrosis. El factor más representativo de recurrencia local es la invasión de márgenes quirúrgicos (14-17).

El pronóstico resulta imprevisible, ya que puede recurrir o metastatizar incluso en ausencia de características de malignidad, y por otro lado los criterios de malignidad se asocian pero no son en sí mismos predictivos de comportamiento agresivo, aunque parece más frecuente cuando se dan (15).

Por tanto se recomienda excisión quirúrgica amplia y seguimiento clínico y radiológico en todos los casos, tanto benignos como malignos. La quimioterapia y la radioterapia han sido aplicadas en casos de resección incompleta o recurrencia, pero su valor es incierto $(4,14)$.

El diagnóstico diferencial no es fácil y con frecuencia siguen siendo confundidos con otras entidades (18). Lesiones benignas que pueden ser confundidas con TFS incluyen histiocitoma fibroso benigno, miofibroblastoma, hemangiopericitoma lipomatoso, lipoma fusocelular e hiperplasia pseudoangiomatosa estromal. En cuanto a su diagnóstico diferencial con neoplasias malignas debemos descartar la presencia de carcinoma metastásico, mesotelioma desmoplástico, dermatofibrosarcoma protuberans, sarcoma epitelioide, leiomiosarcoma, sarcoma miofibroblástico, y liposarcoma indiferenciado $(3,15)$.

\section{BIBLIOGRAFÍA y LECTURAS RECOMENDADAS (*lectura de interés $y^{* *}$ lectura fundamental)}

1. NASCIMENTO, A.G.: "Solitary fibrous tumor: a ubiquitous neoplasm of mesenquymal differentiation". Adv. Anat. Pathol., 3: 388, 1996.

2. KLEMPERER, P.; RABIN, C.B.: "Primary neoplasms of the pleura: A report of five cases". Arch. Pathol., 11: 385,1931 .
**3. GRAADT VAN ROGGEN, J.F.; HOGENDOORN, P.C.W.: "Solitary fibrous tumour: The emerging clinicopathologic spectrum of an entity and its differential diagnosis". Current Diagnostic Pathology, 10: 229, 2004.

4. VALLAT-DECOUVELAERE, A.V.; DRY, S.M.; FLETCHER, C.: "Atypical and malignant solitary fibrous tumors in extratorcic loctions". Am. J. Surg. Pathol., 22: 1501,1998

5. GOODLAD, J.R.; FLETCHER, C.D.M.; "Solitary fibrous tumour arising at unusual sites: Análisis of a series". Histopathology, 19: 1501, 1991.

6. OGURO, S.; TANIMOTO, A.; JINZAKI, M. y cols.: "Imaging findings of solitary fibrous tumor of the prostate: A case report". Mag. Res. Imag., 24: 673, 2006.

7. PACIOS CANTERO, J.C. y cols.: "Tumor fibroso solitario de la próstata”. Actas Urol. Esp., 29: 985, 2005.

*8. GARCÍA TORRELLES, M.; BELTRÁN ARMADA, J.R.; SANTOLAYA GARCÍA, I. y cols.: "Tumor fibroso solitrio de la túnica vaginal”. Arch. Esp. Urol., 59: 186, 2006.

*9. CAFIERO, F.; GIPPONI, M.; PERESSINI, A. y cols.: "Solitary fibrous tumor of the inguinal region: A clinicopathological, light-microscopic, inmunohistochemical, electrón microscopic and flow-cytometric DNA study". Anticancer Res., 21: 4091, 2001.

10. KEMPSON, R.L.; FLETCHER, C.D.M.; EVANS, H.L. y cols.: "Fibrous and myofibroblastic tumors. Tumors of the soft tissues". Washington DC: Armed Forces Institute of Pathology, 52, 2001.

11. HANAU, C.; MIETTTINEN, M.: "Solitary fibrous tumor: histological and inmunohistochemical spectrum of benign and malignant variants presenting at different sites". Hum. Pathol., 26: 440, 1995.

12. BRUNNEMAN, R.B.; RO, J.Y.; ORDÓÑEZ, N.G. y cols.: "Extrapleural solitary fibrous tumor: A clinicopathologic study of 24 cases". Mod. Pathol., 12: 1034, 1999.

*13. WANG, H.; ZHANG, W.; YAN, L. y cols.: "Clinical pathological analysis and inmunohistochemical study of ten solitary fibrous tumors". Chi. Med. J., 115: 1412, 2002.

*14. TRASTOUR, C.; PICHE, M.; BAFGHI, A. y cols.: "Solitary Fibrous tumor of the pelvis". European Journal of Obstetrics \& Gynecology and Reproductive Biology, 124: 254, 2006.

*15. CEBRIÁN GARCÍA, C.; DEL AGUA, C.; FELIPO BERLANGA, F. y cols.: "Tumor fibroso solitario subcutáneo maligno: ¿Pronóstico impredecible? Revista Española de Patología, 36: 215, 2003.

16. YOKOI, T.; TSUZUKI, T.; YATABE, Y. y cols.: "Solitary fibrous tumor: Significance of p53 and CD 43 inmunoreactivity in its malignant transformation". Histopathology, 32: 423, 1998.

17. BROZZETTI, S.; D’ANDREA, N.; LIMITI, M.R. y cols.: "Clinical behaviour of solitary fibrous tumor of the pleura. An inmunohistochemical study". Anticancer Res., 20: 4701, 2000.

**18. XAMBRE, L.; LAGES, R.; CERQUEIRA, M. y cols.: "Tumor fibroso solitario. Dos casos adicionales con implicaciones urológicas”. Actas Urol., 27: 832, 2003. 